\title{
Stowage Planning with Optimal Ballast Water
}

Jia, Beizhen; Fagerholt, Kjetil; Reinhardt, Line; Rytter, Niels Gorm

Published in:

Computational Logistics. ICCL 2020

DOI:

10.1007/978-3-030-59747-4_6

Publication date:

2020

Document Version

Peer reviewed version

Citation for published version (APA):

Jia, B., Fagerholt, K., Reinhardt, L., \& Rytter, N. G. (2020). Stowage Planning with Optimal Ballast Water. In Computational Logistics. ICCL 2020 (pp. 84-100). Springer. Lecture Notes in Computer Science Vol. 12433 https://doi.org/10.1007/978-3-030-59747-4_6

\section{General rights}

Copyright and moral rights for the publications made accessible in the public portal are retained by the authors and/or other copyright owners and it is a condition of accessing publications that users recognise and abide by the legal requirements associated with these rights.

- Users may download and print one copy of any publication from the public portal for the purpose of private study or research.

- You may not further distribute the material or use it for any profit-making activity or commercial gain.

- You may freely distribute the URL identifying the publication in the public portal.

Take down policy

If you believe that this document breaches copyright please contact rucforsk@kb.dk providing details, and we will remove access to the work immediately and investigate your claim. 


\title{
Stowage Planning with Optimal Ballast Water
}

\author{
Beizhen Jia ${ }^{1}$, Kjetil Fagerholt ${ }^{2}$, Line Blander Reinhardt ${ }^{3}$, and Niels Gorm \\ Malý Rytter 4 \\ 1 Aalborg University, A. C. Meyers Vænge 15, 2450 Copenhagen SV, Denmark \\ bj@m-tech.aau.dk \\ 2 Norwegian University of Science and Technology, Trondheim, Norway \\ 3 Roskilde University, Universitetsvej 1, 4000 Roskilde, Denmark \\ 4 University of Southern Denmark, Campusvej 55, 5230 Odense, Denmark
}

\begin{abstract}
Stowage planning is at the essence of a maritime supply chain, especially for short sea Ro-Ro ships. This paper studies stowage optimisation of Ro-Ro ships with a focus on stability constraints and the applicability of models. The paper contributes to short sea Ro-Ro ship stowage in two ways. First, we propose an integrated approach of designing stowage models with the consideration of loading computers. Second, we present a mathematical formulation of the Ro-Ro Ship Stowage Problem with Ballast Water with a discretisation method, to generate an optimal stowage plan which meets stability requirements by means of the weight of cargoes instead of excess ballast water, i.e. excess fuel consumption. Computational tests based on empirical data indicate significant savings and potential of model application in the real world. Preliminary results show $57.69 \%$ ballast water reduction, equivalent to $6.7 \%$ fuel savings and $\mathrm{CO}_{2}$ reduction. Additional tests on instances with various cargo weight distribution and discretisation levels are conducted, and finally, improvements are suggested for further research considerations.
\end{abstract}

Keywords: Stowage optimisation $\cdot$ Ballast water $\cdot$ Maritime transportation · Environmental impact.

\section{Introduction}

Roll-on/Roll-off (Ro-Ro) short sea shipping (SSS) has become one of the most important means in Europe for transportation of passengers and cargoes. Ro-Ro ships carry vehicles and passengers travelling with own journey plans as well as cargo units being trailers, cars, heavy machinery, containers, or anything that goes onto a rolling equipment. Trailers and rolling cargoes are transported either accompanied or unaccompanied by a (truck) driver. Compared to other means of intra-European transport, like for example, container shipping, rail or pure road transport, Ro-Ro SSS has the advantage of being well integrated into the entire cargo supply chains from door to door. Short sea container shipping requires

\footnotetext{
* Supported by ECOPRODIGI.
} 
several modal shifts (road, rail, ship etc.), has longer transit times and low flexibility due to less frequent departures, and implies more document handling in comparison with Ro-Ro SSS.

The European Ro-Ro SSS market is growing [3], but also becoming increasingly competitive with currently approximately 100 short sea operators in Europe [1]. Several Ro-Ro companies have recently expanded their fleet capacity via ordering new mega Ro-Ro ships. One example is DFDS which recently ordered 6 x 6700 lane metre Ro-Ro ships to be delivered to their routes in 2019-2020 [19]. The increase in Ro-Ro tonnage, combined with recent Brexit and Corona virus developments, is likely to impact ship utilisation and rate levels going forward. Also the industry must comply with environmental regulations and International Maritime Organization (IMO)'s 2030 and 2050 targets for greenhouse gas emissions [6]. New ship designs, propulsion technologies and fuel alternatives are in progress to meet long term requirements, but in parallel cost control and energy efficient ship operations will be a strategic priority for existing Ro-Ro operators. A key element will be reduction in fuel consumption and costs on the sea leg via adjusting speed, trim, reduced deadweight etc. To achieve this, it is critical for ships to be stowed optimally before departing from port, which implies maximising cargo load, reducing ballast water intake and thereby deadweight without compromising stability, strength or safety requirements. Ship stowage is a key part of Ro-Ro SSS operations, and it includes a whole set of maritime related sub-processes from when a cargo unit gates into the terminal until when it gets picked up at the destination terminal. It is critical that the entire process is understood well to be able to plan and execute optimal stowage for Ro-Ro ships. In addition to what was mentioned above, high quality stowage planning also ensures efficient load and discharge processes at the terminal, and shortening of the port stay which again enables the ship to slow down and save fuel on the sea leg.

Through interviews with selected terminal managers, stowage planners, ship officers and other relevant stakeholders from one of the largest Ro-Ro shipping companies in Europe, the end-to-end cargo stowage process is defined as a process of a series of cargo-related activities including booking, gate-in, yard positioning at loading port, stowage planning, loading, discharging, yard positioning at destination port and gate-out.

As illustrated in Figure 1, stowage planning acts as the core activity in the process. It takes booking information and cargo arrival status as input to the planning. The booking information offers a list of cargo booked for the departure with detailed cargo information, such as cargo type, transportation unit type, dimensions and weight, well in advance. In addition, cargo arrival status confirms the presence of booked cargo in the terminal on the day, due to the fact that noshow is a common phenomenon in shipping industry. Therefore, before making a stowage plan, the planner needs to know how much of the booked cargo have actually shown up, so that he does not plan stowage for cargo that will never show up and makes timely decision to pull forward optional cargo if the ship's capacity is not fulfilled, thus maximising the space utilisation onboard. 


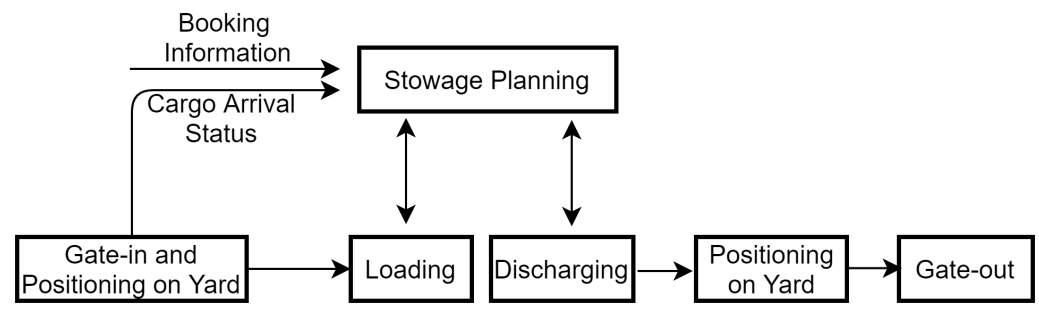

Fig. 1. The Ro-Ro shipping End2end Stowage Process.

Once the load list is updated, a stowage plan is made with consideration of ship stability and cargo characteristics. The stowage plan includes a plan for positions of all cargo to be loaded onboard the ship to ensure a good handling of cargo with regards to special requirements for dangerous goods, refrigerated goods, and goods with lashing needs. The loading operation is conducted according to the stowage plan, and it gets updated if there is any changes happening during the loading operation. Based on the updated stowage plan, the discharge operation is performed at the destination port, and customers can pick up cargo units according to the pick-up time and position in the yard. Finally, customers gate out with their cargo and usually continue road transportation to the next destination.

From above, it becomes visible that stowage planning interacts with all activities happening in the end-to-end cargo stowage process. Hence, it is essential to make a good stowage plan, as it impacts ship utilisation, fuel consumption, safety at sea and the ability to execute load and discharge operations efficiently. Moreover, it can also be used to derive accurate information of when cargo is available for pick-up by customers at the destination port [9].

\section{Literature Review}

Stowage planning of Ro-Ro ships has not attracted the same attention from researchers in operations research and optimisation as has container ships. Øvsteb $\varnothing$ et al. [13] were the first to introduce the Ro-Ro ship stowage problem (RSSP). For a set of mandatory and optional cargoes and a given route with multiple port calls, reflecting the situation of deep sea car carriers, the problem was to determine which additional cargoes to carry and how to stow all carried cargoes on board the ship in order to maximise the profit of the journey. Cargo consisted of a number of homogeneous vehicles. Decks were divided into several lanes which also explained why only rolling moment and vertical forces were constrained in the model for stability considerations. The paper proposed a mixed integer programming (MIP) approach as well as a heuristic algorithm to solve the RSSP. According to the authors, realistic size instances with 5-10 mandatory cargoes could be solved to optimality by MIP, while the heuristic worked better without stability constraints.

Hansen et al. [5] focused on the operational decisions related to the stowage of Ro-Ro ships visiting multiple ports. The paper restricted the stowage problem 
to a single deck and considered it as a special version of a 2-dimensional packing problem with some additional considerations. In addition, it also considered the shifting of cargoes to make an entry/exit path if needed during loading and unloading operations. Several versions of new MIP formulation for the problem were presented with the consideration of reducing the need of shifting. The goal was to stow all mandatory cargoes and as many optional cargoes as possible while trying to avoid shifting. Since it was focused on a single deck, stability constrains were therefore not included in the model. Furthermore, the model used a grid representation of the deck instead of dividing it into several lanes, which the authors thought may restrict finding of good solutions. The paper concluded that the model works well with small-size instances and suggested further research of a faster algorithm for realistic-size problem instances and eventually for not only one single deck but the whole ship.

Following their previous work [5], Hansen et al. [4] presented the stowage plan evaluation problem to determine which vehicles to shift at each port call, in order to minimise the extra time spent on shifting. For a given set of alternative stowage plans, the goal was to find the best plan of all with the minimal shifting time. A shortest path based heuristic was proposed for solving the problem and it showed that solution method was powerful for its fast computing time and high success rate in determining a better plan.

The above mentioned papers were focused on deep sea Ro-Ro ships, such as car carriers that operate globally with multiple port calls on the sailing route. The problems were usually considered with two types of cargoes, mandatory and optional. The objective was therefore focused on revenue related decisions, such as how many optional cargoes could be stowed, less shifting cost, and etc. Stability constraints were simplified and limited for the ease of modelling, and not included at all in the case [5] where only one deck is considered. Such handling of stability constraints might be due to the fact the RSSP with deep sea car carriers is more robust to changes in terms of cargo weight. There is limited variation for car weights. Thus it becomes more relevant for deep sea car carriers to focus on shifting costs along multiple ports on the route in their stowage planning. Nonetheless, when planning stowage for short sea Ro-Ro ships, stability is of utmost importance due to high variance of cargo weights. The difference of cargo weights can have a significant effect on ship's stability in many aspects.

Based on the state-of-art research on Ro-Ro stowage optimisation problem, Puisa [14] proposed three practical improvements, namely ship stability, fire safety and cargo handling efficiency. The author proposed a new grid method to discretise the stowage location onboard for accurate ship stability and strength calculation. Fire safety was ensured by adding additional constraints to high risk cargoes, average headroom and cargo spacing. With the argument that it was not a realistic solution to penalise cargo shifting with a cost as proposed by previous researches, elimination of such was proposed in the paper for a swift loading and unloading operation with multiple port calls. The study included different cargo types with the same weight within a type which might not be the case of containers and trailers for example. The test instance size was small with 
most cargoes being optional. So it is difficult to say the running performance when solving realistic sized problems. The study extended stability calculations with stricter and more constraints. However, without the inclusion of ballast tanks in the calculation, limits for stability constraints should be adjusted to the cases without ballast tanks.

Integration of various operations to improve terminal efficiency has been studied by some researchers in containers shipping, such as ship loading problems where stowage planning is taken into consideration as an input to the model [8][7][10], and stowage planning integrated with the quay crane scheduling [2].

Rethink of the stowage problem. No matter how fast the algorithm works or how much revenue the objective function can achieve, stability is always the prerequisite of a stowage plan. Without it, a ship can not sail. Therefore, it is mandatory to calculate stability for every ship departure to ensure its seaworthiness, which is enforced by IMO. Every ship has a loading computer onboard which connects to sensors that collect all information needed to calculate stability of the ship. Once all cargoes are loaded, ship officers will try to adjust the amount of ballast water in each tank to reach the desirable stability. This usually ends up with ships carrying around with excess ballast water, in other words, excess fuel consumption.

Therefore, the contribution of this paper is twofold - first, to introduce the integrated approach of stowage planning with considerations of loading computers, which has not been studied so far to the authors' knowledge, and second, to include ballast water optimisation in RSSP with the purpose of generating a stowage plan that reduces fuel consumption and at the same time provides a better stability condition that is closer to the loading computer requirements.

\section{$3 \quad$ Integrated Stowage Planning}

Stowage planing for Ro-Ro ships is typically done through a stowage module in combination with onboard loading computer software. A stowage module can be as advanced as stowage optimisation models or as simple as Excel sheets. Loading computers provide deck officers the ability to validate whether a given stowage plan complies with maritime authorities' stress and stability requirements. A ship is required to be seaworthy at any given moment in order for her to sail. At each ship departure, during and after finishing loading, the hull strength and stability of the ship are calculated and if necessary modified by adjusting the amount of water in ballast tanks to meet stability requirements. Currently in the market for Ro-Ro ships, there are several loading computer systems available, such as Kockumation's Loadmaster, NAPA's Loading Computer, Navis's MACS3 and Autoship's Autoload.

The common and traditional approach of designing stowage plans, as illustrated in Fig.2, starts with a stowage module generating an initial stowage plan, optimises if possible and then sends it to the loading computer onboard to check the stability of the plan. If it passes the loading computer's stability requirement, the plan can be executed in the loading process. Otherwise, the ship officer or 
stowage planner manually adjusts the plan by adding ballast water and/or moving cargoes around to achieve desired stability. It is usually the case that the plan does not fulfil the stability requirement from the loading computer, especially when the stowage module provides an optimal plan with bad stability condition to the loading computer. In the case of a stowage model with simplified stability constraints, it may perform excellent in finding optimal solutions according to the objective function. Nonetheless, it may result in undesirable overall performance due to the fact that manual adjustment can be expensive regarding the excess amount of ballast water which is translated to excess fuel consumption.

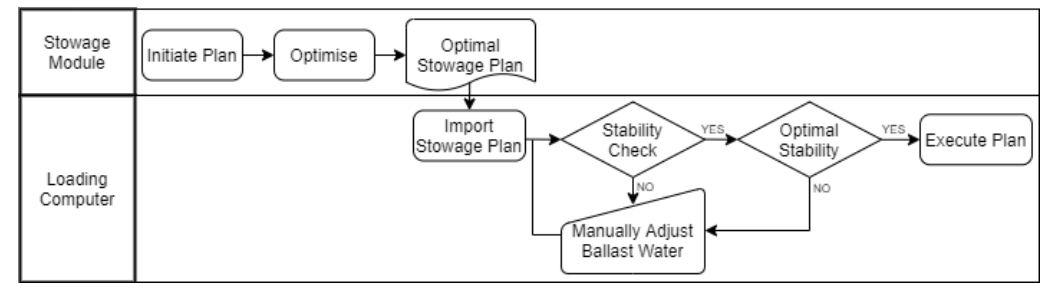

Fig. 2. Traditional stowage planning and interaction with loading computers.

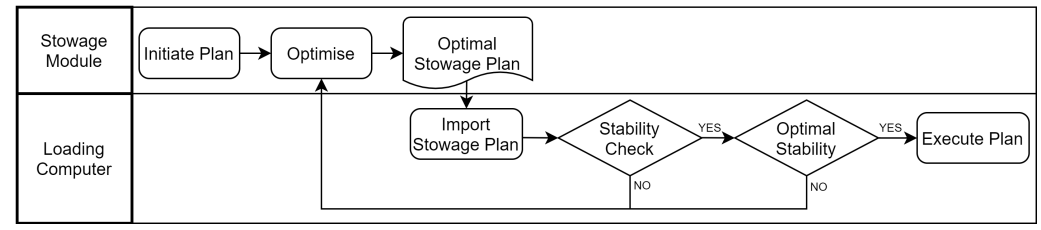

Fig. 3. Envisioned future approach for stowage planning with integration of loading computers.

We propose an integrated approach of stowage planning as decision support, illustrated in Fig. 3. Compared with the traditional approach, the difference is that the loading computer is integrated into the planning phase, meaning that when the optimal plan does not pass stability check, instead of manual adjustment, the information is sent back to the module with additional constraints added to re-optimise and re-generate a new optimal solution. In this approach, there is continuous communication and interaction between the stowage module and the loading computer to improve the plan for it to satisfy stability requirements in the end. The envisioned future approach is automated to the extent that the integration with the loading computer allows. Anyways, these iterations can be expensive, and hence should ideally be eliminated or minimised. Therefore stability constraints should be set as close to reality as possible for the stowage module to generate a good plan subject to a certain objective function while keeping stability within required limits or even optimal stability. In this paper, we focus on designing the stowage model with considerations of the integration with loading computers instead of the whole iterative process, which highly depends on the development of loading computers. 


\section{Ro-Ro Ship Stowage Problem with Ballast Water}

Let us assume that a given Ro-Ro ship transporting two types of cargoes: general trailers (TRAs) and refrigerated trailers (TRARs). TRAs can be loaded anywhere, whereas TRARs can only be loaded at designated slots that have power connection through the ship. The ship has a fixed number of decks with various weight limits. In the case of short sea Ro-Ro ships, the majority of cargoes are standard trailers. For the sake of simplicity, we assume cargoes are homogeneous in dimensions with the same length, width, and height, however, different in weight. All cargoes are mandatory and available at the loading port, unaccompanied by drivers after delivered to the terminal. A number of tug masters are assigned for loading and unloading tasks between ship and shore. Cargoes are loaded onto and discharged from the ship through the main ramp usually located at the aft of the ship. Movement of cargoes within the ship is conducted through narrower ramps located on the side of the ship in between decks. For this characteristic of Ro-Ro ship, cargoes need to be loaded and discharged following precedence relations based on their positions on board.

In order to generate a stowage plan that is more likely to pass stability requirements in the loading computer, it is important to include stability measurements from three dimensions, namely vertical, transverse and longitudinal forces imposed on the ship, measured through metacentric height $(G M)$, heel and trim values as shown in Figure 4. These values are complicated to calculate and are dependent on various factors according to naval architecture [15]. Therefore, they are represented by the composite vertical centre of gravity $(\overline{V C G})$ from the keel $(K G)$, transverse centre of gravity $(\overline{T C G})$ from midship and longitudinal centre of gravity $(\overline{L C G})$ from aft perpendicular to mimic the stability as close to reality as possible.

The vertical distance between composite $\overline{V C G}$ to the metacentre is $G M$, which is calculated by the equation $K G+G M=K M$, where $K M$ is the height of metacentre from keel and can be found in the hydrostatic table from the ship builder. For the simplicity of the model, $K M$ is treated as a constant. $G M$ is one of the most important measurement when it comes to stability. GM is always positive to make sure that ships have the ability to bring themselves back to the upright position. Ship designers usually produce and define a set of values of minimum $G M\left(G M_{\text {min }}\right)$ that meet all intact and damage stability criteria. If the actual $G M$ value is higher than $G M_{\min }$, then in most cases, other stability requirements will also be satisfied [18]. On the other hand, a very large $G M$ meaning that the ship returns to the upright position too fast. At this stage, it has too much stability and becomes stiff, which can cause damage to cargoes and discomfort of crew. Therefore, a maximum $G M\left(G M_{\max }\right)$ value should be enforced as well. Hence, $K G$ should satisfy $K M-G M_{\max } \leq K G \leq$ $K M-G M_{\min }$.

Another two important parameters of stability are $\overline{T C G}$ and $\overline{L C G} \cdot \overline{T C G}$ is an estimation of how much the ship heels, to ensure the ship does not roll too much to one side due to imbalanced heavy load. $\overline{L C G}$ is of similar concept to $\overline{T C G}$ but works in longitudinal direction, and serves as an estimation of trim to 


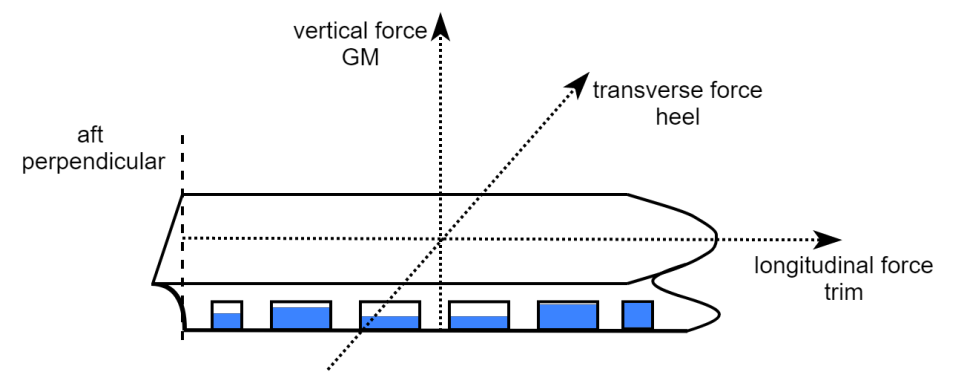

Fig. 4. Ship stability illustration.

make sure the ship does not have a too heavy nose or bottom sitting in the water. Both $\overline{T C G}$ and $\overline{L C G}$ are constrained to a limit range to achieve close-to-zero heel and trim. Note that trim is a more complicated matter which has an impact on fuel consumption. However, it has a non-linear relation to displacement, draught and speed of the ship, hence, trim optimisation is left out in this article.

Ballast tanks are located at the bottom and along both sides of the ship, as illustrated in Figure 4. There are two different types of ballast tanks on board - heeling tanks and regular tanks. Most of the ships have an anti-heeling system which is designed to balance the ship continuously and automatically with heeling tanks to minimise the angle of heel during loading and unloading operations. The total amount of water in all heeling tanks are required to be within a certain range in order to provide sufficient anti-heeling capability when the ship is heeled within a certain range of angles. However if it is beyond the ability of the anti-heeling system, then the amount of water in regular tanks needs to be adjusted to ensure stability. For carrying the same cargo load, the more ballast water a ship carries, the deeper it sits in the water due to the extra deadweight, the more fuel is consumed. In other words, fuel consumption has a positive correlation with the amount of extra ballast water a ship carries.

For a given Ro-Ro ship, transporting a set of cargoes from one loading port to one discharging port, we present an optimisation problem dealing with where to stow each cargo on board so that the ship can sail with a minimal amount of ballast water while still respecting the ship's stability requirements. We consider decisions such as the placement of individual cargo on board with regards to its weight and stowing restrictions, and the amount of ballast water in each tank. In order to integrate with loading computer, we introduce the inclusion of ballast tanks as well as more complete and accurate stability constraints introduced above, in order to achieve overall efficiency of stowage planning with a goal of reducing fuel consumption. We define this problem as Ro-Ro Ship Stowage Problem with Ballast Water (RSSPBW). 


\section{Mathematical Formulation}

We start this section by introducing a list of notation, before presenting the mathematical formulation for the RSSPBW described in Section 4. This formulation contains non-linear stability constraints due to the introduction of ballast water. Therefore, we propose a method of linearising these constraints resulting in a binary integer linear programming formulation.

$\begin{array}{ll}\text { Indices } & \\ c & \text { cargo unit } \\ d & \text { deck } \\ s & \text { slot } \\ i & \text { ballast tank } \\ & \\ \text { Sets } & \text { set of cargo units } \\ \mathcal{C} & \text { subset of refrigerated units } \\ \mathcal{C}^{\mathcal{R}} & \text { set of decks } \\ \mathcal{D} & \text { set of slots } \\ \mathcal{S} & \text { subset of all slots on deck } d \in \mathcal{D} \\ \mathcal{S}_{d} & \text { subset of all refrigerated slots on deck } d \in \mathcal{D} \\ \mathcal{S}_{d}^{\mathcal{R}} & \text { set of ballast tanks } \\ \mathcal{T} & \text { subset of regular ballast tanks } \\ \mathcal{T}^{\mathcal{H}} & \text { subset of heeling ballast tanks } \\ \mathcal{T}^{\mathcal{B}} & \end{array}$

\section{Parameters}

$H^{\max / \min }$

limiting water volume required in all heeling tanks

$T_{i}^{\max }$

$C_{c}^{W}$

$D_{d}^{W, \max }$

$C_{c}^{R}$

$D_{d}^{H}$

$L^{W}$

$L^{V C G}$

$T_{i}^{A o B}$

$T_{i}^{T C G}$

$T_{i}^{L C G}$

$C^{V C G}$

$S_{s}^{V C G / T C G / L C G}$

$K G^{\max / \min }$

$\overline{T C G}^{\max / \min }$

$\overline{L C G}^{\max / \min }$

maximum volume capacity for ballast tank $i$

individual weight of cargo unit $c$

maximum allowable weight on deck $d$

$=1$ if cargo unit $c$ is refrigerated, 0 otherwise

height of deck $d$

lightweight of the ship

VCG of lightship

Area of the base for ballast tank $i$

TCG of ballast tank $i$

LCG of ballast tank $i$

VCG of individual cargo unit

VCG/TCG/LCG of slot $s$

maximum/minimum limiting $K G$ value

maximum/minimum limiting $\overline{T C G}$ value

maximum/minimum limiting $\overline{L C G}$ value

$\rho$

sea water density, unit ton $/ \mathrm{m}^{3}$

\section{Variables}

$x_{c d s}$

$t_{i}$

$K G$

$\overline{T C G}$

$\overline{L C G}$

(binary) $=1$ if cargo $c$ is loaded on deck $d$ at slot $s$ (continuous) the mass of water in ballast tank $i$ composite VCG from keel composite TCG from midship composite LCG from aft perpendicular 


\subsection{Mathematical Formulation}

$$
\begin{aligned}
& \min \sum_{i \in \mathcal{T}^{\mathcal{B}}} t_{i} \\
& \sum_{d \in \mathcal{D}} \sum_{s \in \mathcal{S}_{d}} x_{c d s}=1 \quad c \in \mathcal{C}, c \notin \mathcal{C}^{R} \\
& \sum_{d \in \mathcal{D}} \sum_{s \in \mathcal{S}_{d}^{\mathcal{R}}} C_{c}^{R} x_{c d s}=1, \quad c \in \mathcal{C}^{R} \\
& \sum_{c \in \mathcal{C}} x_{c d s} \leq 1, \quad d \in \mathcal{D}, s \in \mathcal{S}_{d} \\
& \sum_{c \in \mathcal{C}} \sum_{s \in \mathcal{S}_{d}} C_{c}^{W} x_{c d s} \leq D_{d}^{\max }, \quad d \in \mathcal{D} \\
& \rho H^{\min } \leq \sum_{i \in \mathcal{T}_{\mathcal{H}}} t_{i} \leq \rho H^{\max } \\
& K G^{\min } \leq K G \leq K G^{\max } \\
& \overline{T C G}^{\text {min }} \leq \overline{T C G} \leq \overline{T C G}^{\max } \\
& \overline{L C G}^{\text {min }} \leq \overline{L C G} \leq \overline{L C G}^{\text {max }} \\
& x_{c d s} \in\{0,1\}, c \in \mathcal{C}, d \in \mathcal{D}, s \in \mathcal{S} \\
& 0 \leq t_{i} \leq \rho T_{i}^{\max }, i \in \mathcal{T} \\
& K G=\frac{\sum_{c \in \mathcal{C}} \sum_{d \in \mathcal{D}} \sum_{s \in \mathcal{S}_{d}}\left(S_{s}^{V C G}+C_{c}^{V C G}\right) C_{c}^{W} x_{c d s}+L^{W} L^{V C G}+\sum_{i \in \mathcal{T}} t_{i} \frac{t_{i}}{\rho T_{i}^{A o B}}}{\sum_{c \in \mathcal{C}} C_{c}^{W}+L^{W}+\sum_{i \in \mathcal{T}} t_{i}} \\
& \overline{T C G}=\frac{\sum_{i \in \mathcal{T}} t_{i} T_{i}^{T C G}+\sum_{c \in \mathcal{C}} \sum_{d \in \mathcal{D}} \sum_{s \in \mathcal{S}_{d}} x_{c d s} C_{c}^{W} S_{s}^{T C G}+L^{W} L^{T C G}}{\sum_{c \in \mathcal{C}} C_{c}^{W}+L^{W}+\sum_{i \in \mathcal{T}} t_{i}} \\
& \overline{L C G}=\frac{\sum_{i \in \mathcal{T}} t_{i} T_{i}^{L C G}+\sum_{c \in \mathcal{C}} \sum_{d \in \mathcal{D}} \sum_{s \in \mathcal{S}_{d}} x_{c d s} C_{c}^{W} S_{s}^{L C G}+L^{W} L^{L C G}}{\sum_{c \in \mathcal{C}} C_{c}^{W}+L^{W}+\sum_{i \in \mathcal{T}} t_{i}}
\end{aligned}
$$

The objective of the model (1) minimises the total amount of ballast water carried onboard a ship in order to reduce the fuel consumption caused by excess ballast water. Constraints (2) and (3) ensure that each cargo is loaded exactly once at a slot for general cargo and refrigerated cargo respectively. Whereas constraints (4) make sure that each slot will only have at most one cargo loaded. For ship safety and stability, constraints (5) make sure that the total weight of cargoes loaded on each deck does not exceed the maximum weight limit per deck. Constraint (6) keeps the total amount of water in heeling tanks within a safe margin so that the tanks have sufficient capability to heel the ship. Lastly, vertical, transverse and longitudinal stability calculations are presented in equation (12), (13) and (14), and are constrained through constraints (7), (8) and (9), respectively. Due to the inclusion of ballast tanks and the amount of water inside as decision variables, $V C G$ of ballast tanks becomes a function of the decision variables as well. For a given ballast tank, its $V C G$ depends on the volume of water inside, and its shape, or its area of base if the tank is shaped vertically straight. The 
model assumes the latter, as also shown in Equation (12). Lastly, decision variables are bounded by (10) for binary indicator $x_{c d s}$, and by (11) for both types of ballast tanks, whose upper limits are taken from ship builders.

\subsection{Linearisation}

As can be observed that constraints (7), (8) and (9) are non-linear when substituted with equation (12), (13) and (14), respectively, not only due to the division but also the quadratic function of decision variables $t_{i}$ in equation (12).

To eliminate the division existing in all three constraints, we simply multiply each constraint with its lower fraction, which is the sum of all weights including the ship itself. It is naturally positive, hence does not have any impact on the signs of the inequalities. It is however trickier when it comes to linearising the quadratic function in equation (12) - the product of the amount of water in the ballast tank and its vertical centre of gravity which is again determined by the amount of water whether the tank empty, full or in between. We propose a discretisation method using the following additional notations listed. In the discretisation method, we divide each tank $i$ into several filling levels denoted by a set of discrete points $k \in \mathcal{K}_{i}$ and use binary variables $y_{i k}$ to indicate whether the tank is filled to a certain level $k$. Each point or filling level corresponds to an amount of water $T_{i k}^{V O L}$ and a VCG value $T_{i k}^{V C G}$ when tank $i$ is filled to the level $k$.

$\begin{array}{lll}\text { Indices } & k & \text { discretisation point, fill level of ballast tank } \\ \text { Sets } & \mathcal{K}_{i} & \text { set of discretisation point for ballast tank } i \\ \text { Parameters } & T_{i k}^{V O L} & \text { volume of ballast tank } i \text { if filled at level } k \\ & T_{i k}^{V C G} & \text { VCG of ballast tank } b \text { if filled at level } k \\ \text { Variables } & y_{i k} & \text { (binary) }=1 \text { if ballast tank } i \text { is filled at level } k\end{array}$

An example of the discretisation method is illustrated in Figure 5. Let us look at one of the ballast tanks on board, tank $i$, which is located right above the keel. The maximum amount of water tank $i$ can carry is $100 \mathrm{~m}^{3}$ and its maximum VCG value is 10 metres. The tank is divided into three levels denoted by a set of points $\mathcal{K}_{i}=\{0,1,2\}$. At filling level $k=0$, tank $i$ is empty and therefore its corresponding $V C G$ is 0 metres. A half filled tank $i$ corresponds to a filling level of $k=1$, with a $V C G$ of 5 metres. Lastly a filling level of $k=2$ meaning that the tank is full with $100 \mathrm{~m}^{3}$ of ballast water inside and a $V C G$ of 10 metres. In the case illustrated here, the tank is filled to level $k=1$, represented by binary variables $y_{i 2}=1$, and $y_{i 0}=y_{i 1}=0$. As mentioned above, the discretised tank values corresponding to filling level $k=2$ are $50 \mathrm{~m}^{3}$ of ballast water and a $V C G$ of 5 metres. Therefore, the gravity moment of the tank $i$ becomes the following linear calculation:

$$
\sum_{k \in \mathcal{K}_{i}} \rho T_{i k}^{V O L} T_{i k}^{V C G} y_{i k}=\rho(0 \times 0 \times 0+50 \times 5 \times 1+100 \times 10 \times 0)=250 \rho(t-m)
$$

The method represents the decision variables $t_{i}$, and their corresponding $V C G$ in a discrete manner and replaces them in the original formulation in Section 5.1 so that the quadratic product can be linearised. The amount in ballast tank $t_{i}$, their corresponding $V C G$, and their gravity moment are now represented as

$$
t_{i}=\sum_{k \in \mathcal{K}_{i}} \rho T_{i k}^{V O L} y_{i k} \quad i \in \mathcal{T}
$$




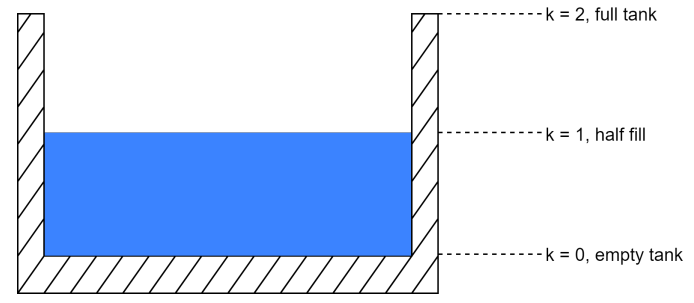

Fig. 5. Tank discretisation.

$$
\begin{array}{rlrl}
T_{i}^{V C G} & =\sum_{k \in \mathcal{K}_{i}} T_{i k}^{V C G} y_{i k} & i \in \mathcal{T} \\
t_{i} T^{V C G}=\sum_{i \in \mathcal{K}_{i}} \rho T_{i k}^{V O L} T_{i k}^{V C G} y_{i k} & i \in \mathcal{T}
\end{array}
$$

Constraint (7) is then linearised and rewritten as the following:

$$
\begin{aligned}
& K G^{\text {min }}\left(\sum_{c \in \mathcal{C}} C_{c}^{W}+L^{W}+\sum_{i \in \mathcal{T}} \sum_{i \in \mathcal{K}_{i}} \rho T_{i k}^{V O L} y_{i k}\right) \leq \\
& \sum_{c \in \mathcal{C}} \sum_{d \in \mathcal{D}} \sum_{s \in \mathcal{S}_{d}}\left(S_{s}^{V C G}+C_{c}^{V C G}\right) C_{c}^{W} x_{c d s}+L^{W} L^{V C G}+\sum_{i \in \mathcal{T}} \sum_{k \in \mathcal{K}_{i}} \rho T_{i k}^{V O L} T_{i k}^{V C G} y_{i k} \\
& \leq K G^{\max }\left(\sum_{c \in \mathcal{C}} C_{c}^{W}+L^{W}+\sum_{i \in \mathcal{T}} \sum_{i \in \mathcal{K}_{i}} \rho T_{i k}^{V O L} y_{i k}\right)
\end{aligned}
$$

By substituting $t_{i}$ with formula (15) in all other appearance in the original formulation and constraint (7) with 18, a new linearised formulation of RSSPBW is presented.

\section{Computational Results}

We collected empirical data from one of the largest short sea Ro-Ro shipping companies in Europe. One departure has been selected as the benchmark in this study due to the complexity of working with the loading computer. The departure was from Vlaardingen, the Netherlands to Immingham, the UK. The ship deployed for the route has a capacity of 4076 lane meters with two heeling tanks and 20 regular ballast tanks in various sizes. Empirical data regarding the departure consisted of a stowage plan carried out by the dispatcher and the crew, a list of cargo information, and a file from the loading computer on board containing the ship's condition upon departure. Limits for the stability constraints were roughly estimated based on zero trim condition with the help of an naval architect working with the loading computer. For this specific ship, the limits applied in the model are [11, 12.5], [87.83, 93.61] and [-0.5, 0.5] for $K G$, $L C G$ and $T C G$ respectively. The linearised RSSPBW was run in Julia with Gurobi optimiser on a window laptop with Intel(R) Core(TM) i7-7820HQ CPU @ $2.90 \mathrm{GHz}$ and 16.0 GB RAM. The optimal solution was found in 65 seconds with an input size of 251 cargoes with a total weight of 6976 tons and 10 discretisation levels of ballast tanks.

Preliminary results on ballast water savings and stability conditions are shown in Table 1. The original stowage plan (orig.) collected from the empirical data carried 3448.5 tons of ballast water, whereas the optimal solution (opt.) from the linearised 


\begin{tabular}{|ll|l|l|l|l|}
\hline Parameters & unit & orig. & opt. & opt.s & opt.orig.s \\
\hline Ballast water & amount (ton) & 3448.5 & 911.4 & 1458.9 & 2115.5 \\
& saving in (ton/\%) & $0.0 / 0.00$ & $2537.1 / 73.57 \%$ & $1989.6 / 57.69 \%$ & $1333.0 / 38.65 \%$ \\
\hline Stability & GM (metre) & 1.51 & 1.43 & 1.23 & 1.28 \\
from loading & Trim (metre) & $0.00 \mathrm{f}$ & $1.93 \mathrm{a}$ & $0.91 \mathrm{a}$ & $0.06 \mathrm{a}$ \\
computer & Heel (degree) & $0.1 \mathrm{~s}$ & $1.8 \mathrm{~s}$ & $1.5 \mathrm{~s}$ & $0.4 \mathrm{~s}$ \\
\hline Fuel consumption & amount (ton) & 10404.7 & 9520.3 & 9707.6 & 9946.9 \\
*annual & saving (ton/\%) & $0.0 / 0.00$ & $884.4 / 8.5 \%$ & $697.1 / 6.7 \%$ & $457.8 / 4.4 \%$ \\
& monetary saving & $\$ 0$ & $\$ 502339$ & $\$ 395953$ & $\$ 260030$ \\
\hline $\mathrm{CO}_{2}$ impact & emission (ton) & 32412.0 & 29657.0 & 30240.4 & 30985.9 \\
$*$ annual & reduction (ton) & 0 & 2755.0 & 2171.6 & 1426.1 \\
\hline
\end{tabular}

Table 1: Ballast and fuel consumption saving results based on real stowage plan and sailing condition.

RSSPBW minimised the amount of ballast water down to 911.4 tons with a saving of 2537.1 tons, accounted for almost $75 \%$ of the original amount. However, the optimal solution provided a plan that is heavily trimmed by the aft with a risky GM and not approved by the loading computer due to the stability requirement. For the sake of the performance and comparison, we improve the optimal solution by manually adjusting the amount of ballast water on board to meet the loading computer's requirement (opt.s). The result when the ship is within stability is still astonishing - over half the original ballast intake was cut off. Moreover, we also improved the plan a step further by adjusting the ship to match the stability condition in the original stowage plan with a close-to-even trim and heel (opt.orig.s). Once again, we are still able to save $38.65 \%$, which is equivalent to an amount of 1333 tons saving of the original amount of ballast water. Furthermore, to translate ballast savings into fuel consumption savings and $\mathrm{CO}_{2}$ reductions, we roughly estimated the fuel consumption by using admiralty coefficient [12], average fuel consumption and $\mathrm{CO}_{2}$ emission of a ro-ro ship close to the empirical ship [11], route distance [16] and an average bunker price of $\$ 568$ per metric ton for MGO in Rotterdam in 2019 [17]. For one ship sailing on the selected route with a daily departure, the annual savings in fuel consumption are 697.1 and 457.8 tons for the cases where stability requirements are satisfied. Their respective monetary savings are $\$ 348,550$ and $\$ 228,903$. Moreover, a saving in fuel consumption has a positive impact on our environment. As presented in the table, $\mathrm{CO}_{2}$ emission can be reduced significantly with an amount of 2171.6 tons. Note that the savings in "opt.s" and "opt.orig.s" are only minimal since they were based on manual improvement from a non-expert.

The preliminary results show that the RSSPBW has a significant benefit on ballast savings with stability constraints and integration with loading computer, even though it is based on only one departure. Setting the right limits for stability constraints in the linearised RSSPBW is a complicated matter involving one to master the knowledge of navel architecture. A better set of limits will definitely contribute to a ship condition closer to ideal stability. Furthermore, a larger set of discretisation points provides a higher level of granularity for the filling levels and in turn improves the flexibility of the model satisfying the stability constraints. However, it might be expensive. In order to evaluate the impact of different discretisation levels on the running time, we performed the following tests. Based on the above empirical load list, we generated 10 instances with different cargo weight distribution and run them against three discretisation levels $\left|\mathcal{K}_{i}\right|=\{10,50,100\} \forall i$ to examine the performance variation, displayed in Table 2 . In 


\begin{tabular}{|l||l|l|l|l||l|l|l|l|l|l|l|l|}
\hline \multirow{2}{*}{ Instance } & \% of cargo weigh between & \multicolumn{3}{|c|}{$\left|\mathcal{K}_{i}\right|=10$} & \multicolumn{2}{|c|}{$\left|\mathcal{K}_{i}\right|=50$} & \multicolumn{2}{|c|}{$\left|\mathcal{K}_{i}\right|=100$} \\
& $5-15$ & $15-25$ & $25-35$ & $35-45$ & $t$ & $t^{\prime}$ & $t$ & $t^{\prime}$ & $t$ & $t^{\prime}$ \\
\hline inst1 & 25 & 25 & 25 & 25 & 2.63 & 2.82 & 2.21 & 2.30 & 2.16 & 2.20 \\
inst2 & 20 & 30 & 30 & 20 & 2.78 & 2.86 & 2.46 & 2.50 & 3.26 & 3.33 \\
inst3 & 30 & 20 & 20 & 30 & 1.72 & 1.77 & 1.68 & 1.74 & 3.26 & 3.28 \\
inst4 & 10 & 40 & 40 & 10 & 2.27 & 2.32 & 2.99 & 3.05 & 3.17 & 3.28 \\
inst5 & 40 & 10 & 10 & 40 & 2.27 & 2.28 & 2.38 & 2.42 & 2.74 & 2.77 \\
inst6 & 10 & 20 & 30 & 40 & 110.38 & 9.28 & 31.66 & 18.65 & 37.32 & 8.44 \\
inst7 & 40 & 30 & 20 & 10 & 1.70 & 1.62 & 2.53 & 2.45 & 2.72 & 2.60 \\
inst8 & 50 & 50 & 0 & 0 & 2.30 & 2.18 & 1.67 & 1.61 & 2.48 & 2.39 \\
inst9 & 0 & 50 & 50 & 0 & 16.77 & 4.70 & 38.03 & 15.26 & 9.64 & 2.19 \\
inst10 & 0 & 0 & 50 & 50 & 63.48 & 8.52 & 17.52 & 8.20 & 17.2 & 3.49 \\
\hline
\end{tabular}

Table 2: Test instances and results.

addition, we compare the running time when it solves to optimality $(t)$ with the running time when it is terminated by $1 \%$ gap $\left(t^{\prime}\right)$, equivalent to less than 2 tons ballast water.

Most instances can be solved to optimality within 4 seconds regardless of the discretisation levels. For cases that are difficult for the model to find the optimal solution, such as inst6, inst9 and inst10, a larger discretisation level can significantly improve the running time as assumed above, but at the same time a too large discretisation level can be costly as indicated in the test results of inst6, where the running time was improved significantly from 110 s to 31 s from a discretisation level of 10 to 50, while with $\left|\mathcal{K}_{i}\right|$ increased from 50 to 100 , the performance dropped. No obvious pattern has been found on the correlation between discretisation level and running time. There are several other deciding factors such as the strictness of stability constraints, the granularity of tanks and the cargo distribution as well. However, for cases where optimality is difficult to achieve, getting close to the lower bound with $1 \%$ gap can be done at a much lower computational cost. This indicates the ease of implementing the model in the real world, namely fast running time providing a close-to-optimum solution.

\section{Conclusion and Discussion}

This paper analyses the problem of stowage planning in short sea Ro-Ro shipping and proposes an integrated approach to model and solve stowage and stability problems. The new approach requires better formulation of stability constraints and the inclusion of ballast water compared to previous methods. The idea is to generate an optimal stowage plan which uses the weight of cargoes to satisfy stability requirements instead of using excess ballast water which is translated into excess fuel consumption. The paper defines a Ro-Ro ship stowage problem with ballast water and presents a quadratic mathematical formulation with the objective to optimise the amount of ballast water onboard. A discretisation method is applied to linearise the quadratic constraints due to the introduction of ballast tanks. The linearised model is then tested against empirical data collected from the collaborating company. Computational results on the selected departure indicate significant potential for ballast savings, showing the relevance of the model's application in the real world. Furthermore, additional tests on instances with various cargo weight distributions and discretisation levels are conducted, and results show no significant correlation among the factors. 
Our preliminary study result from this research clearly indicates the industry potential of our integrated stowage approach and model which delivered between $4.4 \%$ and $6.7 \%$ of savings in fuel consumption and emissions. Due to the complexity of the problem, some details were simplified and compromised compared to reality, which can be further improved by a more complete and better formulated set of constraints. For example, additional deadweight elements, such as storage and fuel tanks can be included for a more accurate stability calculation; free surface movement can be implemented by penalising partially filled tanks; trim optimisation can be added since it has an obvious impact on fuel consumption etc. In addition, other discretisation methods such as piecewise linear functions might improve the solution without significantly increase computational costs. Another aspect which we suggest for future research is to analyse the robustness of the model, subject to changes of cargo amounts, mix and weight. As mentioned, the unpredictability of cargo amounts and composition, makes it difficult to apply our model in daily processes without making it more robust. Lastly, even though the majority of cargo is homogeneous in dimensions, cargo in reality differ in sizes compared to standard trailers. Future research and models for stowage planning can therefore also improve practical relevance by including this aspect.

\section{References}

1. European transport maps, https://www.europeantransportmaps.com/map/roroferry/operator

2. Azevedo, A.T., de Salles Neto, L.L., Chaves, A.A., Moretti, A.C.: Solving the 3d stowage planning problem integrated with the quay crane scheduling problem by representation by rules and genetic algorithm. Applied Soft Computing 65, 495516 (2018). https://doi.org/10.1016/j.asoc.2018.01.006

3. Eurostat: Maritime transport statistics - short sea shipping of goods, https://ec.europa.eu/eurostat/statistics-explained/index.php/ Maritime_transport_statistics_-_short_sea_shipping_of_goods

4. Hansen, J.R., Fagerholt, K., Stålhane, M.: A shortest path heuristic for evaluating the quality of stowage plans in roll-on roll-off liner shipping. In: Bektas, T. (ed.) Computational logistics, LNCS sublibrary: SL1 - Theoretical computer science and general issues, vol. 10572, pp. 351-365. Springer, Cham, Switzerland (2017)

5. Hansen, J.R., Hukkelberg, I., Fagerholt, K., Stålhane, M., Rakke, J.G.: 2d-packing with an application to stowage in roll-on roll-off liner shipping. In: Paias, A., Ruthmair, M., Voß, S. (eds.) Computational Logistics, Lecture Notes in Computer Science, vol. 9855, pp. 35-49. Springer International Publishing, Cham (2016)

6. IMO: Greenhouse gas emissions, http://www.imo.org/en/OurWork/Environment/ PollutionPrevention/AirPollution/Pages/GHG-Emissions.aspx

7. Iris, Ç., Christensen, J., Pacino, D., Ropke, S.: Flexible ship loading problem with transfer vehicle assignment and scheduling. Transportation Research Part B: Methodological 111, 113-134 (2018). https://doi.org/10.1016/j.trb.2018.03.009

8. Ji, M., Guo, W., Zhu, H., Yang, Y.: Optimization of loading sequence and rehandling strategy for multi-quay crane operations in container terminals. Transportation Research Part E: Logistics and Transportation Review 80, 1-19 (2015). https://doi.org/10.1016/j.tre.2015.05.004

9. Jia, B., Rytter, N.G.M., Reinhardt, L.B., Haulot, G., Billesø, M.B.: Estimating discharge time of cargo units - a case of ro-ro shipping. In: Paternina-Arboleda, C., Voss, S. (eds.) Computational logistics, LNCS sublibrary: SL1 - Theoretical 
computer science and general issues, vol. 11756, pp. 122-135. Springer, Cham, Switzerland (2019)

10. Jovanovic, R., Tanaka, S., Nishi, T., Voß, S.: A grasp approach for solving the blocks relocation problem with stowage plan. Flexible Services and Manufacturing Journal 31(3), 702-729 (2019). https://doi.org/10.1007/s10696-018-9320-3

11. LIPASTO: Average emissions and energy use of a roro and ropax ship in 2016, http://lipasto.vtt.fi/yksikkopaastot/tavaraliikennee/vesiliikennee/ roroe.htm

12. Man Diesel \& Turbo: Basic principles of ship propulsion, https: //spain.mandieselturbo.com/docs/librariesprovider10/sistemaspropulsivos-marinos/basic-principles-of-ship-propulsion.pdf?sfvrsn=2

13. Øvstebø, B.O., Hvattum, L.M., Fagerholt, K.: Optimization of stowage plans for roro ships. Computers \& Operations Research 38(10), 1425-1434 (2011)

14. Puisa, R.: Optimal stowage on ro-ro decks for efficiency and safety. Journal of Marine Engineering \& Technology pp. 1-17 (2018)

15. Rhodes, M.A.: Ship stability for mates. Seamanship International Ltd, Glasgow (2003)

16. Searoutes: Immingham - vlaardingen distance, https://www.searoutes.com/ routing/4294967447/4294973215

17. Ship and Bunker: Rotterdam bunker prices, https://shipandbunker.com/prices/ emea/nwe/nl-rtm-rotterdam\#MGO

18. Wärtsilä: Wärtsilä encyclopedia of marine technology: Curve of minimum operational metacentric height gm, https://www.wartsila.com/encyclopedia/term/ curve-of-minimum-operational-metacentric-height-gm

19. World Maritime News: Dfds names its largest roro ship - offshore energy (2019), https://www.offshore-energy.biz/dfds-names-its-largest-roro-ship/ 